\title{
Induced visual movement as nonveridical resolution of displacement ambiguity
}

\author{
R. H. DAY \\ Monash University, Victoria 3168, Australia
}

\begin{abstract}
Following the proposals made by Kinchla, it is argued that induced movement is a nonveridical resolution of stimulus ambiguity. The ambiguity derives from an identity between displacement of one element relative to another and displacement of the second relative to the first in a featureless field at velocities below the threshold for subject-relative movement. In such conditions, which element actually moves is perceptually unresolvable and veridical judgments therefore accord with chance. When a stationary field is introduced, perception is veridical, but when the field moves with the moving element, perception is nonveridical, i.e., induced movement of the stationary element and induced stationariness of the moving element occurs. The results from three experiments supported this interpretation and showed also that movement velocities above the subject-relative threshold contribute to the resolution of ambiguity.
\end{abstract}

Induced movement is the apparent movement of a stationary element in the opposite direction to the real movement of another element in an otherwise dark or featureless field. The stimulus arrangement commonly used to demonstrate induced movement consists of a small stationary target enclosed within a moving frame. However, the effect occurs with a variety of stimulus arrays, including simply two points of light, one moving and the other stationary (Duncker, 1929; Mack, Fisher, \& Fendrich, 1975), a stationary field of radiating lines surrounded concentrically by a similarly patterned, rotating annulus (Duncker, 1929), and a small stationary grating within a larger moving grating (Day \& Dickinson, 1977; Over \& Lovegrove, 1973).

Two explanations of induced movement have recently been proposed. Brosgole (1968) has argued that it can be regarded as a special case of the Roelofs effect (Roelofs, 1935), a shift in the egocentric localization of a point in the direction of a larger element in the visual field. Brosgole suggested that, as the frame enclosing a small stationary target moves, the egocentric localization of the point shifts with the changing position of the frame. While this explanation is plausible for the conventional display consisting of a stationary point in a laterally moving frame, it is less so for other arrangements. For example, a stationary circular pattern appears to rotate when a concentric patterned annulus moves (Duncker, 1929). It seems unlikely that the apparent egocentric position of the stationary target would shift under such conditions. Furthermore, movement of a

The assistance of Mr. R. G. Dickinson in the collection and analysis of data is gratefully acknowledged. stationary point can be induced by real movement of a similar point adjacent to it (Duncker, 1929; Kinchla, 1971; Mack, Fisher, \& Fendrich, 1975). The Roelofs effect involves a relatively large and dominant element towards which the egocentric shift occurs. It is unclear why one small point rather than another identical to it should determine egocentric direction.

An explanation in terms of inhibitory interaction between feature detectors in the visual system has been proposed by Over and Lovegrove (1973). This explanation is based largely on an observed reduction in the strength of induced movement when the wavelength characteristics of stationary target and moving field were different. Recently, this reduction has been shown to be specific to particular stimulus arrangements involving a moving grating framed in a stationary aperture. No such reduction occurs with the conventional stationary point and moving frame arrangement or a pattern composed of gratings in which the field moves as a whole (Day \& Dickinson, 1977).

An alternative explanation that the three experiments reported here were designed to test can be derived jointly from a quantitative model of movement perception developed by Kinchla and his associates (Kinchla, 1971, 1976; Kinchla \& Allan, 1969) and a notion of perceptual resolution of stimulus equivocality (Day, 1969, 1972). Kinchla distinguishes between perception of absolute movement and perception of relative movement. The first refers to movement of an object in a featureless field relative to the observer himself and is also called subjectrelative movement (Shaffer \& Wallach, 1966). The second refers to movement of one object relative to another and is also called object-relative movement. ${ }^{1}$ Kinchla has pointed out that for the case of two small 
targets in a homogeneous field, one moving and the other stationary, the observer could be quite accurate in discriminating change in separation between the two but not in discriminating which of the two is displaced. He has also found (Kinchla, 1971) that for object-relative movement perception, the separation between the targets cannot exceed about $15^{\circ}$ in visual angle under normal conditions of viewing.

The threshold for subject-relative movement is considerably higher than for object-relative movement (Aubert, 1886; Shaffer \& Wallach, 1966). Thus for a two-target array in an otherwise featureless field with the velocity of the moving target for a particular exposure duration below the subjectrelative threshold and with a separation of less than $15^{\circ}$ between them, movement of one target would result in a relative displacement perceptually identical to that resulting from movement of the other in the opposite direction, i.e., the stimulus movement would be perceptually equivocal. ${ }^{2}$ It follows, therefore, that over a number of trials veridical judgments of movement and stationariness would be expected to accord with chance. That this is so was confirmed by Mack, Fisher, and Fendrich (1975). Now, if a stationary, enclosing frame were introduced, movement and stationariness of the targets would, of course, be relative to it and judgments of both would thus be expected to be veridical. That is, the ambiguous displacement of the targets would be veridically resolved. If, on the other hand, the frame were moved in the same direction and at the same velocity as the moving target, that target and frame would be relatively stationary and the stationary target relatively displaced in the opposite direction. Assuming that the frame continues to be the reference, judgments of movement and stationariness would now be expected to be nonveridical. Furthermore, if velocity were increased so that it achieved and exceeded the subject-relative threshold, i.e., movement of the target relative to the observer himself, veridical perception of movement would be expected to be increasingly probable, since movement of elements relative to the observer himself would be progressively easier to discriminate.

In summary, an ambiguous and, therefore, perceptually unresolvable stimulus array consisting of identical stationary and moving elements can be expected to be resolved veridically as real movement and stationariness nonveridically as induced movement according as a stationary or a moving field is present. It follows that when, as is common, the display consists of a single, stationary target and a moving field, it is the field-like properties which serve to resolve the ambiguity. Thus, a critical issue yet to be fully explored is the nature of those stimulus properties which distinguish target from field. This issue is taken up briefly again below.
The three experiments reported here were designed to test two hypotheses derived from the explanation of induced movement proposed here. The first, which was tested in Experiment 1, was that movement of one target relative to another in an otherwise featureless field at a velocity below the subject-relative threshold is ambiguous and is resolved veridically or nonveridically by a stationary or a similarly moving field, respectively. The second, which was tested in Experiments 2 and 3, was that as the velocity of the moving elements (target and field) is increased above the subject-relative threshold nonveridical resolution (induced movement) occurs less frequently. ${ }^{3}$

\section{EXPERIMENT 1}

In the first experiment, subjects judged which of two targets was moving when the stationary and moving target were presented alone, enclosed in a stationary frame, and enclosed in the same frame moving in the same direction and at the same velocity as the moving target. Velocity was below the subjectrelative threshold throughout. For the first condition, it was expected that the stimulus would be unresolvable in terms of which of the two targets was moving and that correct judgments would therefore approximate chance. In the second condition, it was expected that the stimulus array would be resolved veridically and that most judgments would be correct. For the third, the expectation was for nonveridical resolution, i.e., induced movement and stationariness of the moving and stationary target, respectively.

\section{Method}

Subjects. There were 10 paid undergraduate and graduate subjects, 5 men and 5 women. None were familiar with the stimulus array, although some had participated in experiments on induced movement earlier.

Apparatus. The main features of the apparatus, consisting of a moving slide driven by a motor operating through a rack and pinion arrangement and a stationary slide carrying the moving and stationary stimulus elements, respectively, have already been described (Day \& Dickinson, 1977; Day, Dickinson \& Forster, 1976). Subjects viewed the stimulus arrays consisting of two targets alone, one above the other (condition TT), the two targets surrounded by the stationary frame (TTS), or the two surrounded by the moving frame (TTM) from a distance of $287 \mathrm{~cm}$. At this distance, $5 \mathrm{~cm}$ subtends a visual angle of $1^{\circ}$. The targets were $12^{\prime}$ in diameter with a vertical separation between centers of $24^{\prime}$. The frame was $4^{\circ}$ vertically and $6^{\circ}$ laterally in outside dimensions, and the frame itself was $18^{\prime}$ wide. The luminance of all elements was $.5 \mathrm{~cd} / \mathrm{m}^{2}$. One target, with or without the frame, could be moved laterally at $6 \% / \mathrm{sec}$, a speed below the subject-relative threshold. “ The exposure period of $2 \mathrm{sec}$ during which the stimulus array was visible was automatically controlled. During the session, the subject was seated at a table. Only the stimulus array was visible in the dark room.

Procedure. The subject's task throughout was to indicate whether the upper or lower target was moving during a 2 -sec exposure. There were 24 such trials, 8 for each of the conditions 
TT, TTS, and TTM. During 4 trials in each condition, the upper target was moved twice to the left and twice to the right, and during the other 4 , the lower target was moved similarly. The order of the 24 trials was separately randomized for each subject. Before each session, taped instructions were played to the subject and questions about procedure encouraged and answered. At the beginning of each trial, the experimenter signaled "ready," after which the stimulus array was visible for $2 \mathrm{sec}$ with the targets initially in vertical alignment and, for conditions TTS and TTM, centered in the frame. The intertrial interval varied between about 10 and $15 \mathrm{sec}$. The score for each condition was the number of correct judgments of which target moved during the 2-sec exposure. Thus the maximum score was 8 .

The method selected to test the outcome for each condition in each of the three experiments was to compare it with the chance outcome, i.e., 4 , using the $t$ test for uncorrelated means. Since for the three experiments there were nine independent tests, the $\alpha$ level was set at .005 . The probability of rejecting the null hypothesis when it was true in one or more of these tests is .04 (Winer, 1962, p. 199).

\section{Results}

It was expected that for condition TT the number of correct judgments would not exceed chance, i.e., 4 correct, and that for conditions TTS and TTM there would be an increase and a decrease, respectively, in the number of correct judgments.

The mean numbers of correct judgments for each of the three stimulus conditions are shown together with their standard deviations in Table 1 . It can be seen that the number correct corresponded closely to chance for condition TT and shifted markedly in the direction of veridicality in condition TTS and of nonveridicality in TTM. Three $t$ tests confirmed that for TT the mean was not significantly different from chance, $\mathrm{t}(9)=1.05, \mathrm{p}>.05$, but that for TTS and TTM there was a significant increase and decrease, respectively, in the number correct, $\mathrm{t}(9)=24.38$, $\mathrm{p}<.005, \mathrm{t}(9)=28.61, \mathrm{p}<.005$. It can be noted also that, for TT, individual scores ranged between 3 and 6 , for TTS, between 7 and 8, and for TTM, between 0 and 1 .

Table 1

Means and Standard Deviations of Number of Veridical Judgments of Which of Two Targets was Moving

\begin{tabular}{llrrr}
\hline & & \multicolumn{3}{c}{ Stimulus Conditions } \\
\cline { 3 - 5 } & & TT & TTS & TTM \\
\hline \multirow{2}{*}{ Experiment 1} & Mean & 4.40 & 7.70 & .20 \\
& SD & 1.17 & .48 & .42 \\
Experiment 2 & Mean & 5.60 & 8.00 & .90 \\
& SD & 1.35 & 0.00 & .99 \\
Experiment 3 & Mean & 6.90 & 8.00 & 2.30 \\
& SD & .57 & 0.00 & 1.83 \\
\hline
\end{tabular}

Note-TT, two targets alone in a featureless field; TTS, two targets surrounded by a stationary frame; TTM, two targets surrounded by a moving frame. Maximum score $=8$, chance $=4$, $N=10$ for each experiment.
Taken together, the means and ranges for the three conditions are clear in showing that a stationary and a moving field serve to resolve an equivocal stimulus situation, respectively, in terms of the veridical state of affairs and a nonveridical state in which a stationary target seems to move (induced movement) and a moving target seems to be stationary (induced stationariness).

\section{EXPERIMENTS 2 AND 3}

Except for stimulus velocities and subjects, the second and third experiments were the same as the first. They can conveniently be described together. In Experiment 2, the velocity of the moving parts of the stimulus array was slightly above the threshold for subject-relative movement and in Experiment 3 clearly above it. It was expected that because of the greater velocities than in Experiment 1 there would be an increase in the number of correct judgments in conditions TT and TTM, i.e., that induced movement would be less frequent, and that this trend would increase between Experiments 2 and 3. For condition TTS, no change in outcome compared with Experiment 1 was expected, since the maximum number of correct judgments had virtually been achieved.

\section{Method}

Subjects. Two new groups of 10 paid undergraduates participated. Each consisted of 4 men and 6 women.

Apparatus and Procedure. In Experiment 2, the velocity of the moving target and frame was $18^{\prime} / \mathrm{sec}$, about $5^{\prime} / \mathrm{sec}$ above the subject-relative threshold, and in Experiment 3, it was $30^{\prime} / \mathrm{sec}$, about $17^{\prime}$ above the threshold. Apart from these changes, the apparatus and procedure were the same as in the first experiment.

\section{Results}

The mean numbers of veridical judgments for the three conditions are shown for the two experiments in Table 1 together with their standard deviations.

It can be seen that, for Experiment 2, the number of correct judgments for condition TT was greater than 4 , for condition TTS markedly greater, and for condition TTM markedly less. Statistical analysis of the means using the same procedure as in Experiment 1 showed that the difference between the mean for TT and that expected by chance did not achieve an acceptable level of significance, $t(9)=3.75$, $\mathrm{p}>.005$, whereas the differences for TTS and TTM did, $\mathrm{t}(9)=\infty, \mathrm{p}<.005, \mathrm{t}(9)=9.90, \mathrm{p}<.005$. For TT the range of scores was 4-7, for TTS all scores were 8, and for TTM the range was 0-3.

In Experiment 3, the number of correct judgments for condition TT was again greater than 4 , for condition TTS maximally greater, and for condition TTM less. Statistical analysis showed that the difference between the mean for TT and chance expectancy was 
significant, $\mathrm{t}(9)=16.09, \mathrm{p}<.005$, as was that for TTS, $t(9)=\infty, p<.005$. The difference between the mean for condition TTM and chance, however, did not achieve an acceptable level, $\mathrm{t}(9)=2.94, \mathrm{p}>.005$.

Thus, in Experiment 2, with a stimulus velocity slightly in excess of the subject-relative threshold, the mean score for condition TT did not depart acceptably from chance, but in Experiment 3, with the velocity further above the threshold, the score for this condition moved in the direction of veridicality. In both experiments, the mean scores for condition TTS were maximally veridical. In Experiment 2, the mean for condition TTM differed from chance in the direction of nonveridicality, but that in Experiment 3 did not.

\section{DISCUSSION}

The results from the three experiments can be briefly summarized as follows. When one of two identical targets in a dark field moved with a velocity below the threshold for subject-relative movement, correct judgments of which one moved did not exceed chance, thus confirming the prediction made by Kinchla (1971) and the results reported by Mack, Fisher, and Fendrich (1975). When the targets were enclosed in a stationary frame, judgments were almost entirely correct, i.e., they accorded with the veridical state of affairs. When, however, the targets were enclosed in the same frame moving in the same manner as one of them, judgments were almost entirely wrong, indicating that perceived movement and stationariness were induced in the stationary and moving target respectively. However, when the velocity of stimulus elements for the 2 -sec exposure was above the subject-relative threshold by $5^{\prime} / \mathrm{sec}$, nonveridical effects were slightly less frequent and, when by $17^{\prime} / \mathrm{sec}$, markedly less frequent. This result obtained for the two targets alone (TT) and the two in the moving frame (TTM).

Four issues from these results warrant further discussion: the occurrence of veridical perception with a stationary frame, induced stationariness with a moving frame, the role of objective (field) and egocentric references, and the stimulus properties which distinguish field from target.

The view can be taken that veridical resolution of displacement ambiguity with a stationary field is so obvious as to be trivial. However, to do so would overlook the point that the results from this condition emphasize the role of relative movement as the basis of perceived movement when velocity is below the subject-relative threshold and when, as Kinchla (1971) has pointed out, the elements fall within an angular compass of about $15^{\circ}$ or less. In condition TTS, observers perceived the targets as moving or stationary according as they were moving or station- ary relative to the frame, thereby resolving the ambiguity of displacement when the two targets alone were visible. In condition TTM, they continued to do so. Thus, induced movement and stationariness for velocities below the subject-relative threshold represent relative movement and relative stationariness with the frame the reference.

By using two targets, one stationary and the other moving in similar fashion to the frame, the limiting case of induced stationariness has been demonstrated. Again, this observation is not trivial. The perceived stationariness of a target moving with the frame serves also to stress that with movement velocity below the subject-relative threshold what is perceived as stationary is that which is relatively stationary in respect to the reference field.

The results from Experiments 2 and 3 are clear in showing that when the velocity of movement of target and field exceeds the subject-relative threshold nonveridical effects, i.e., induced movement and induced stationariness, are less frequent. At these velocities, movement of target and frame relative to the observer himself can be perceived. It can be presumed that, under these conditions, the observer himself together with the frame serve as references for perceived movement and stationariness. However, these separate references must give rise to perceptual conflict when the frame is moving. When the actually moving and stationary target are, respectively, stationary and moving relative to the frame, they are moving and stationary relative to the observer. The data from Experiments 2 and 3 indicate that when velocity is well above subject-relative threshold the egocentric reference is more salient than when it is slightly above. Whether this trend continues for higher velocities is yet to be determined. Incidentally, this conclusion accords well with Gogel's $(1974,1977)$ view that conflicting absolute and relative (i.e., subject-relative and object-relative) cues contribute to the perception of motion in a graded manner. It seems from the results reported here that the velocity of movement of stimulus elements is a main determinant of which reference, egocentric or field, is involved in resolving displacement ambiguity.

The last issue concerns what specifies a reference field and distinguishes it from a target. With two targets alone, either can serve as a reference for the other when velocity is below the subject-relative threshold. With a small target and an enclosing frame, the latter invariably serves as the reference field. Duncker (1929) contended that in the twotarget arrangement it is the fixated point which appears to move. Presumably, therefore, he regarded the nonfixated point as the reference. While the results from an earlier study by Thelin (1927) support this contention, those from a recent investigation by Mack, Fisher, and Fendrich (1975) clearly do not. 
Nevertheless, it is to be noted that Thelin found that interactions between fixation, intensity, and real movement determine what is perceived to move and remain stationary when the two elements are similar in size and shape. So far there has been no systematic study of the role of enclosure, intensity size patterning, and other variables in the perceptual determination of reference field and target for velocities beinw the subject-relative threshold. The outcome of the experiments described here identify this issue as central for a complete understanding of induced movement and stationariness.

Thus, at stimulus velocities below the subjectrelative threshold, induced movement and induced stationariness represent the perceptual resolution of an ambiguous displacement in terms of relative stationariness and movement in respect to a moving field as is implied by Kinchla (1971). In this sense, induced movement and stationariness are no different from the veridical resolution in respect to a stationary field. At velocities in excess of the subjectrelative threshold the egocentric reference, i.e., the observer himself, is also involved in the resolution of the ambiguity inherent in the displacement of one object relative to another. In conclusion, it can be noted that this view is not unlike that outlined by Gogel (1977), who has argued that relative and absolute cues contribute to the perception of various environmental events, including object movement.

\section{REFERENCES}

Aubert, H. Die Bewegungsempfindung. Archiv für die Gesamte Physiologie, 1886, 39. 347-370.

Brosgole, L. An analysis of induced motion. Acta Psychologica, $1968,28,1-44$.

Brown, R. H., \& Conkin, J. E. The lower threshold of visible movement as a function of exposure-time. American Journal of Psychology, 1954, 67, 104-110.

DAY, R. H. Human perception. Sydney: Wiley, 1969.

DAY, R. H. Visual spatial illusions: A general explanation. Science, 1972, 175, 1335-1340.

Day, R. H., \& Dickinson, R. G. Absence of colorselectivity in Duncker-type induced visual movement. Perception \& Psychophysics, 1977, 22, 313-320.

Day, R. H., Dickinson, R. G., \& Forster, K. I. Induced subject-relative movement: Persistence of apparent movement of stationary point after removal of inducing stimulus. Perception \& Psychophysics, 1976, 19, 510-517.

DUNCKER, K. Über Induzierte Bewegung (Ein Beitrag zur Theorie optisch Wahrgenommener Bewegung. Psychologische Forschung, 1929, 12, 180-259.

Gogel, W. C. Relative motion and the adjacency principle. Quarterly Joumal of Experimental Psychology, 1974, 26. 425-437.
GOGEL, W. C. The metric of visual space. In W. Epstein (Ed.), Stability and constancy in visual perception: Mechanisms and processes. New York: Wiley, 1977. Pp. 129-181.

Kinchla, R. A. Visual movement perception: A comparison of absolute and relative movement discrimination. Perception \& Psychophysics, 1971, 9, 165-171.

Kinchla, R. A. A psychophysical model of visual movement perception. In R. Monty \& J. Senders (Eds.), Eye movements and psychological processes. New York: Erlbaum, 1976.

Kinchla, R. A.. \& Allan, L. G. A theory of visual movement perception. Psychological Review, 1969, 76, 537.558.

MAck, A., Fisher, C. B., \& Fendrich, R. A re-examination of two-point induced movement. Perception \& Psychophysics, $1975,17,273-276$.

Over, R.. \& Lovegrove, W. Color-selectivity in simultaneous motion contrast. Perception \& Psychophysics, 1973, 14, 445-448.

Roelofs, C. O. Optische Lokalisation. Archiv für Augenheilkunde, 1935, 109, 395-415.

Shaffer, O., \& Wallach, H. Extent of motion thresholds under subject-relative and objective-relative conditions. Perception \& Psychophysics, 1966, 1, 447-451.

Thelin, E. Perception of relative visual motion. Journal of Experimental Psychology, 1927, 10. 321-349.

WinER, E. J. Statistical principles in experimental design. (2nd ed.) New York: McGraw-Hill, 1962.

\section{NOTES}

1. The terms "absolute and relative movement" and "subjectrelative and object-relative movement," respectively, are synonymous. Here the latter two terms are preferred, since in each case the movement is relative either to the observer himself (subjectrelative) or to another object in the visual field (object-relative).

2. Since subject-relative movement involves a change of position of the stimulus object in the dark relative to the observer himself, the detectability of movement varies with both stimulus velocity and stimulus exposure duration. That is, the change of position can be detected in a short exposure if velocity is high but only in a relatively long exposure if it is low (Brown \& Conklin, 1954). In the experiments reported here, exposure duration was always $2 \mathrm{sec}$.

3. Of course, the three experiments could have been included as three conditions of one experiment. This was not possible, since no allowance had been made for varying the speed of the moving elements, apart from that of changing the gearbox. For this reason, three separate experiments were conducted.

4. Brown and Conklin (1954) found that for a 1.97-sec exposure the velocity threshold was $12.18^{\prime} / \mathrm{sec}$, and Mack, Fisher, and Fendrich (1975) found that for a 2 -sec exposure it was $13.21^{\prime} / \mathrm{sec}$. The latter also found that the range of velocity thresholds for a 2 -sec exposure was $8.89^{\prime}$ to $16.5^{\prime} / \mathrm{sec}$. Therefore, the speed of $6 \%$ sec during a 2-sec exposure in Experiment 1 can be assumed to be below the subject-relative movement threshold.

(Received for publication June 13, 1977; revision accepted November 30,1977.) 